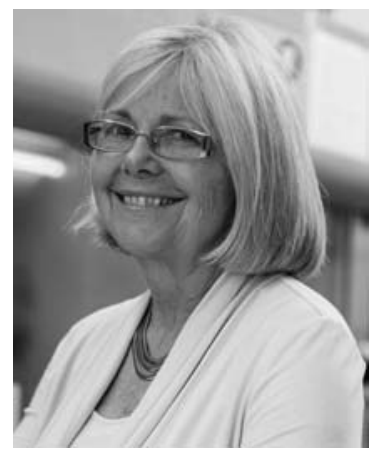

\title{
A Conversation with Joan Brugge
}

\author{
INTERVIEWER: RICHARD SEVER \\ Assistant Director, Cold Spring Harbor Laboratory Press
}

Joan Brugge is Director of the Ludwig Center and Chair of the Department of Cell Biology at Harvard Medical School.

Richard Sever: The first time you came to Cold Spring Harbor was 1974.

Dr. Brugge: That actually wasn't for a meeting. It was to learn how to do a procedure that was novel at the time, and Cold Spring Harbor was one of the only places that did it. It was a hybridization technique called $\mathrm{C}_{0} \mathrm{t}$ analysis. I spent a week at the laboratory of Joe Sambrook and learned how to do it. My first meeting was probably the RNA tumor virus meeting when I moved here in '79.

Richard Sever: You've worked on so many things: the original identification of Src, EGFR (epidermal growth factor receptor) signaling, integrins, metabolism, the microenvironment of apoptosis. An old friend told me that this broad background meant that at one point you were appointed as the commissioner of the Oncogene Olympics at a meeting. Is that true? In refereeing, was it? Apparently, there was "Team Cytoplasm" and "Team Nucleus"?

Dr. Brugge: Yes, exactly. The meeting was so intense that I thought it would be great to have a break; a short break was scheduled in the afternoons, but everybody roamed around. They didn't know what to do, so I thought of an idea for something we could do that would allow us to "bond" and at the same time have a lot of fun-this became the Oncogene Olympics. We had many different events - soccer, softball, tennis - and we broke up into the teams for those people working on either nuclear or cytoplasmic oncogenes. Everyone enjoyed it so much that it became a tradition. It was lots of fun.

Richard Sever: Presumably, you were in "Team Cytoplasm."

Dr. Brugge: Definitely. I stayed away from the nucleus for many years.

Richard Sever: Your recent work remains very much in the cytoplasm, particularly with respect to high-grade ovarian cancer. Can you say why that's such an important cancer to look at, and what the challenges are?
Dr. Brugge: High-grade serous ovarian cancer is one of the most recalcitrant types of cancer. One problem is that the disease isn't detected until generally it's at stage 3 and 4 , and the prognosis for patients at those stages is poor. Their 5-year survival is somewhere around $10 \%$ or $20 \%$, and basically the treatments haven't changed for the last 20 years. Treatment's generally chemotherapy; the first-line treatment is a combination of a platinum- and a taxane-based drug. Eventually, all the tumor cells become resistant, so they basically throw every type of chemotherapy they can at the tumors. Patients go through a cycle of remission, relapse, remission, relapse, over and over, and eventually, the patient succumbs.

Richard Sever: It seemed like there was a lot of information that you learned from the DNA sequencing of tumors from TCGA (The Cancer Genome Atlas). What were the things that you picked up?

Dr. Brugge: The sequence didn't reveal any obvious "smoking gun" that we could go after right away. There weren't mutations in EGFR or ALK (anaplastic lymphoma kinase) or BRAF, or any of the other oncogenes that have drugs that are directed against them, but it did reveal some interesting things. One is that almost every single tumor had a mutation in the tumor suppressor p53, and it looks like that's the first event. That seems to trigger the initiation of the cancer. Additionally, there weren't any other mutations that were found at high frequency, but there were an enormous number of copy-number alterations.

The investigators that worked on the sequencing information were able to discern a few pathways that looked interesting for therapeutic intervention. One is that $\sim 50 \%$ of the tumors had defects in some component of homologous recombination-mediated repair of DNA damage. Those tumors are predicted to be more sensitive to PARP (poly-ADP ribose polymerase) inhibitors, and PARP inhibitors are actually used in the clinic for patients that have tumors that are defective in DNA repair. Unfortunately, it's the same story as with chemo: There's initial

(C) 2016 Brugge. This article is distributed under the terms of the Creative Commons Attribution License, which permits unrestricted reuse and redistribution provided that the original author and source are credited. 
success, but then they undergo remission and relapse. They're trying to figure out what to do about that.

They also noticed that there were common alterations in multiple components of two other pathways: the phosphoinositide 3-kinase (PI3K)/AKT/mTOR pathway, and the RAS/ERK (extracellular-signal-regulated kinase) pathway. About $50 \%$ of the tumors had an amplification or deletion in regulators of those pathways, but then if you looked at single-copy deletion or amplification, they were in a huge percentage of tumors. It raised the question whether slight amplification of an oncogene coupled with slight or one-copy loss of a negative regulator of that pathway would be sufficient to activate the pathway.

Richard Sever: You were essentially seeing PI3K/AKT or ERK pathway signatures in this tumor?

Dr. Brugge: Yes, in terms of the alterations. Along with the genome information from the TCGA study, they also published proteomics data using the RPPA (reversephase protein array) platform that Gordon Mills developed at MD Anderson. That provided strong support that both of these pathways are activated. The phosphoprotein markers that are indicative of pathway activation were enriched in $\sim 70 \%-75 \%$ of the tumors. That's what made us feel that it would be worth exploring these two pathways to see if there would be any therapeutic potential.

Richard Sever: What was the next stage in looking at the consequences of activation of the PI3K pathway?

Dr. Brugge: We decided to use tumor cell samples from patient-derived xenografts (PDXs) that had been developed at Dana-Farber. These match the representation of genomic alterations and pathway activation very nicely, relative to the human tumor data. They grow in mice, we harvest them, and then we have a pipeline for analysis so that we can get as reproducible data as possible. They're cultured exactly the same way for the exact number of days. We found a 10- to 15 -fold variation in their sensitivity to PI3K/mTOR inhibitors. However, what we really found was that while inhibition of these pathways caused a cessation of proliferation, it didn't induce cell death.

We wanted to understand that. We had previous data that suggested there was an adaptive response, like a rewiring of pathways, for the cells to adapt to inhibition of this central pathway that regulates self-survival. Originally, we would have expected there to be an induction of death, but we found there's also this massive upregulation of signaling pathways that provide survival protection. So anti-apoptotic pathways were activated significantly, like Bcl-2 (B-cell lymphoma 2) protein.

Richard Sever: Both pro- and anti-apoptotic pathways went up?

Dr. Brugge: Right. A new analysis by Gordon Mills includes twelve different pro- and anti-apoptotic proteins. They were almost all up-regulated. It wasn't just a survival response; there was also a response to inhibition of this survival pathway, which basically leads to a new balance. Pro-apoptotic mechanisms are up-regulated, but at the same time, there's an adaptive response to counter that, so the cells have to reach a new equilibrium with higher levels of both pro- and anti-apoptotic proteins. That suggested that if we could inhibit the antiapoptotic proteins, it would unleash the elevated activity of the pro-apoptotic proteins to induce cell death.

Richard Sever: It's like the cells have one foot on the gas and one on the brake, and if you could kick the foot off the brake then you'd be okay.

Dr. Brugge: That's what we found. When we combined the Bcl-2/Bcl-xL (B-cell lymphoma, extra-large) combined inhibitor from AbbVie together with PI3K/mTOR inhibitor from Genentech, we got really nice synergistic death on all but a few of the models. There were three models that were much less sensitive, where we didn't see synergy with the Bcl-2/xL inhibitor. A bio-engineer in our lab, Ioannis Zervantonakis, did a systems-level regression analysis. He was able to predict which components of these pro- and anti-apoptotic proteins would be the best predictors for the response to the PI3K/combined inhibition. It worked out beautifully. The predictions made from his analysis were validated completely with the inhibition studies and with proteomic studies. For example, in those three PDX models that were the least sensitive, it was predicted that two other anti-apoptotic proteins, Mcl-1 (induced myeloid leukemia cell differentiation protein) and XIAP, were elevated and were furthermore predicted to correlate with resistance. Indeed, if we inhibited either one of those we got a very dramatic inhibition of survival in those models.

Richard Sever: So it really was a targeted therapy.

Dr. Brugge: Yes, but it goes a step further. The original inhibitor we used was a Bcl-2/xL combined inhibitor, but the regression analysis indicated that $\mathrm{xL}$ was much more predictive. We got separate inhibitors from AbbVie that were specific for Bcl-xL or Bcl-2, and found exactly that. The Bcl-xL inhibitor had great efficacy combining with the PI3K inhibitors, but Bcl-2 inhibitors basically didn't have any activity. We were able to parse out which of the components of the anti-apoptotic program are critical in different models. It looks like they will be good biomarkers if the therapy goes to the clinic.

Richard Sever: That was on the AKT side. You talked about this other pathway, the ERK/MAP-kinase pathway. Were you getting a similar regulation of these apoptosis regulators?

Dr. Brugge: Definitely. The ERK pathway had previously been shown to suppress expression of a very dominant, potent pro-apoptotic called BIM (Bcl-2-like protein 11). When we looked at the RPPA data, there were three different groups of the PDX models: one where ERK was high and BIM was low (which you'd predict), one where ERK was low and BIM was high, and two that were low for both. We suspected that by inhibiting ERK in the models that weren't already high for BIM we could 
up-regulate BIM and sensitize the cells, and that's exactly what happened. Those models in which ERK inhibition led to an increase in BIM were very sensitive to the combination of Bcl-2/xL and ERK or MEK (mitogenactivated protein kinase kinase) inhibition, but the others were not.

Richard Sever: MEK being the protein upstream of ERK.

Dr. Brugge: Right, MEK inhibitor will block the ERK pathway. Again, those models where we didn't see any synergy were the ones that had high Mcl-1, and where we used the Mcl-1 inhibitor combined with the MEK inhibitor we got beautiful synergy. Basically, the same PDX models that were sensitive to PI3K were sensitive to ERK. We found that PI3K inhibitor was inhibiting ERK, so essentially we saw the same activity because the PI3K was upstream of the ERK. When we inhibited PI3K, we inhibited ERK as well.

We've been talking to AbbVie and Genentech about clinical translation of this. They're most interested in a
MEK inhibitor because they have less overall toxicity, and they think it would be easier to combine with the Bcl-2/xL inhibitor. We're setting up to do preclinical studies now to focus on that combination.

Richard Sever: Do you think that we're potentially at an inflection point that's equivalent to the triple cocktail moment with HIV?

Dr. Brugge: I think that's what we're likely going to have to work toward. It's not straightforward because of the complications in clinical trials. Generally, you can just add one at a time, and so you have to do layered trials to look at the addition of a second drug to the first one, and then yet another one. It's not going to be straightforward, but unless immunotherapy can clean up all of those resistant tumor cells from the initial therapies, then we're going to have to get better at killing, which is going to involve more inhibitors. We're hopeful that the immune system will do that. Those are orthogonal therapies so it might be more straightforward than to hit yet another signaling pathway or something. 


\section{$\$_{\text {CSH\& }}^{\infty}$ Cold Spring Harbor Symposia SYMPOSIA}

\section{A Conversation with Joan Brugge}

Cold Spring Harb Symp Quant Biol 2016 81: 301-303 originally published online April 12, 2017 Access the most recent version at doi:10.1101/sqb.2016.81.033035

Creative This article is distributed under the terms of the

Commons http://creativecommons.org/licenses/by/4.0/, which permits unrestricted

License reuse and redistribution provided that the original author and source are credited.

Email Alerting Receive free email alerts when new articles cite this article - sign up in Service the box at the top right corner of the article or click here. 